\title{
ANÁLISIS DE LA MORTALIDAD EXPÓSITA DE LA INCLUSA DE TOLEDO Y SUS CONDICIONANTES (1900-1930)
}

\author{
Noelia M. Martín-Espinosa \\ EU Enfermería y Fisioterapia de Toledo (UCLM) \\ Noelia.Martin@uclm.es \\ Rafael Villena Espinosa \\ Facultad de Humanidades de Toledo (UCLM) \\ Rafael.VEspinosa@uclm.es \\ Ana I. Cobo-Cuenca \\ EU Enfermería y Fisioterapia de Toledo (UCLM) \\ Analsabel.Cobo@uclm.es
}

Recibido: 3 enero 2014; Aceptado: 1 abril 2015.

Cómo citar este artículo/Citation: Martín-Espinosa, Noelia M.; Rafael Villena Espinosa y Ana I. Cobo-Cuenca (2016), "Análisis de la mortalidad expósita de la Inclusa de Toledo y sus condicionantes (1900-1930)", Asclepio 68 (1): p130 doi: http://dx.doi.org/10.3989/ asclepio.2016.09

RESUMEN: El objetivo de este trabajo es analizar la mortalidad expósita y sus causas en una cohorte de niños abandonados en la inclusa toledana, aquellos que nacieron en la Maternidad aneja, como grupo homogéneo que partía de unas condiciones de alumbramiento similares y fueron institucionalizados al nacer. Ello permite comparar esta mortalidad expósita con la de otras inclusas españolas y con la mortalidad poblacional, a la vez que explica los distintos factores que pudieron condicionarla.

PALABRAS CLAVE: Mortalidad infantil; Lactancia materna; Nodrizas; Niños expósitos; Inclusas.

\section{ANALYSIS OF THE MORTALITY EXPERIENCE AMONG THE ABANDONED CHILDREN IN THE FOUNDLING HOSPITAL OF TOLEDO AND ITS DETERMINANTS (1900-1930)}

ABSTRACT: The aim of this work is to analyze the mortality and its causes in the abandoned children of the Children's home of Toledo, who were born in the Maternity House, because it was a homogeneous group which had the same conditions in their delivery and they were abandoned at the moment of their birth. It allows us to compare the mortality of this group of foundlings with the mortality of the general population and with the mortality of those abandoned in other Charity Institutions. This paper explains the different factors which could determine the mortality.

KEY WORDS: Infant mortality; Breastfeeding; Wet-nurses; Abandoned children; Foundlings. 


\section{INTRODUCCIÓN}

Acercarse a la realidad de cualquier inclusa española desde el siglo XVI supone dar con unas cifras de mortalidad tan elevadas que resulta casi inverosímil el objetivo de amparo y protección de los niños abandonados que estas instituciones decían cumplir. Parece, más bien, que se quisiese posponer el momento de una muerte casi segura que sucedería igual si se dejaba al recién nacido abandonado a la intemperie o en el torno de uno de los centros de acogida. La sociedad prefirió la última modalidad porque al menos se intentaba hacer algo por salvar a esos hijos fruto de la miseria, de las relaciones ilegítimas ${ }^{1}$ o de ambas cosas a la vez; y que, rechazados por sus progenitores, quedaban al cuidado de instituciones en donde rara vez se les podría proporcionar un futuro digno. Numerosos estudios de historia social y demográfica analizan este fenómeno, centrados mayormente en los siglos

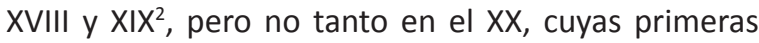
décadas analizaremos en las siguientes páginas.

Resulta complicado atribuir esas muertes a una única causa y más bien debemos pensar en la confluencia de diversos factores: gestaciones complejas por la situación personal de las madres, deficiente situación higiénico-sanitaria de las inclusas y riesgos asociados a una alimentación inadecuada, debida la escasez de amas y a la propensión de cruzar infecciones al compartirlas. También habría que considerar el tiempo de permanencia en la institución, las condiciones de vida de la familia de acogida o la combinación de esta actividad lactante con otras faenas, como las agrarias que, sin duda, interferirían en el cuidado del pequeño.

La lactancia materna fue considerada la única forma segura de alimentar a los recién nacidos hasta, al menos, el primer tercio del siglo XX, pues aunque se conocía y usaba la alimentación artificial desde la segunda mitad del XIX, solo empezó a considerarse una opción recomendable para los pequeños décadas más tarde, dada su alta mortalidad vinculada ${ }^{3}$.

Esto obligaba a las inclusas a la contratación de amas internas y externas para procurar alimentar a los recién nacidos abandonados con leche materna, aunque no todos los centros lo lograban de forma exclusiva ${ }^{4}$. El nodrizaje fue muy cuestionado por higienistas, puericultores y pensadores contemporáneos del fenómeno, pues consideraban que las nodrizas rurales tenían pocos conocimientos sobre cuidar a los pequeños y utilizaban todo tipo de artimañas para obtener un rendimiento económico de esta actividad ${ }^{5}$.
En general, las investigaciones nacionales e internacionales ${ }^{6}$ sobre lactancia asalariada le confieren un papel determinante en las altas tasas de mortalidad entre los niños criados con este sistema, porque entienden que el cuidado prestado a los pequeños era insuficiente $y$, simplemente, devenían en un instrumento para mejorar la economía familiar. Así queda reflejado en varios estudios sobre inclusas españolas, como el de la granadina (de La Fuente, 2000), en la que «el niño de la Casa-Cuna es para el ama un instrumento con el que puede ganar dinero, un negocio, aunque sea mísero... La muerte del expósito afecta muy poco -mejor nada- al ama [...]». Fernández Ugarte apunta en una dirección similar para el caso de la inclusa salmantina a comienzos del siglo XVIII, pero atribuye parte de la responsabilidad del fracaso del modelo de crianza a los bajos salarios que recibían estas mujeres y a la falta de afecto con el niño que amamantaban y al que, a fin de cuentas, consideraban un medio para ganar algo de dinero (Fernández, 1988).

¿Podría afirmase que el sistema de alimentación y crianza en las inclusas españolas conducía a la muerte temprana de los expósitos en vez de a su supervivencia? Algo de cierto puede haber en ello, pero es necesario matizar conclusiones de ese calado, puesto que resulta difícil calibrar el grado de responsabilidad de las amas en la mortalidad de los pequeños, cuyas trayectorias vitales estaban determinadas por múltiples eventos adversos que, relacionados, quizás procuren una explicación más precisa de los fallecimientos. De hecho, algunas investigaciones sugieren una visión holística del nodrizaje. Es el caso del estudio sobre las amas de cría en el reino de Murcia - siglos XVII y XVII- en el que se analizan varios de los factores que debieron influir en la alta mortalidad: el transporte desde la localidad de origen hasta la inclusa, la escasez de amas internas, el tiempo de permanencia en la casa-cuna hasta que salían con un ama externa y, finalmente, también el origen del ama de cría, que determinaría en buena medida las condiciones de vida que le esperaban al recién nacido ${ }^{7}$ (Chacón, Elgarrista y Fresneda, 1987).

El objetivo de nuestra investigación es estudiar la mortalidad de los niños expósitos en la inclusa de Toledo, nacidos en la Maternidad aneja porque sus madres habían pasado allí algún tiempo previo a dar a luz para ocultar su embarazo ilegítimo, con la finalidad de abandonarlos al nacer. Hemos vinculado la mortalidad de los pequeños al ámbito en el que fueron lactados, presentando tasas generales de mortalidad y otras específicas como mortalidad por grupos de edad 
y causas. Con ello pretendemos obtener una visión global de los distintos factores externos que pudieron condicionar esa mortalidad temprana.

El estudio se basa en los libros de lactancias y destetes de los Establecimientos Reunidos de San Pedro Mártir (centro benéfico integrado por la Casa de Maternidad, la Casa-Cuna, el Asilo de Huérfanos y el de Ancianos Pobres) entre los años 1900 y 1930. En ellos se recogen de forma individualizada los registros de cada uno de los niños nacidos en la Maternidad hasta cumplir los cinco años de vida y cesar su cuidado remunerado. El seguimiento (fecha de nacimiento, fallecimiento, lugar y causa) es posible porque cada niño quedaba inscrito en el citado libro con un número de registro que se mantenía invariable durante los cinco años mencionados. En total, 924 expósitos durante los treinta años de análisis. Tanto los libros, como otra documentación relativa al régimen interno y funcionamiento del centro asistencial, se conservan en el Archivo de la Diputación Provincial de Toledo, sección Beneficencia, en donde los hemos consultado.

\section{EL NODRIZAJE O DE CÓMO ALIMENTAR A LOS NIÑOS}

La mayor parte de las inclusas europeas y americanas usaban la lactancia materna para alimentar a los recién nacidos abandonados en ellas hasta que su edad les permitía tolerar otros alimentos. Este sistema suponía la contratación de nodrizas, mujeres con leche materna suficiente que alimentaban a los residentes a cambio de una retribución mensual previamente pactada. Unas pocas amas residían en la propia institución y eran las encargadas de lactar a los pequeños desde el momento del abandono hasta que se les alojaba en el hogar de una nodriza externa en alguna localidad, a veces muy alejada de la inclusa. Cuando las nodrizas escaseaban, recurrían a otras formas de alimentación entre las que destacaba el uso de leche de vaca o cabra y las papillas de harina y agua, aunque raramente resultaron exitosas y, más bien, eran las responsables de la mortalidad prematura de niños cuyo organismo no estaba adaptado para digerir este tipo de sustancias. En efecto, era el modelo seguido en la inclusa de Toledo, según consta en un informe emitido a petición de la Diputación de Zaragoza y en el que se justifica el uso de lactancia artificial - parece que con malos resultados en el pasado- solo en casos extremos de acúmulo de niños o falta de amas ${ }^{8}$.

El mercado de nodrizas externas se nutría de mujeres campesinas, residentes en zonas rurales y cuya pobreza les abocaba a aceptar los exiguos salarios que les ofrecía la institución. Los riesgos eran muchos, in- cluso la posibilidad de contraer enfermedades como la sífilis, pero la necesidad era mayor y ese salario podía constituir un aporte fundamental para las menguadas economías familiares ${ }^{9}$.

En torno a la lactancia asalariada se fue gestando una cierta preocupación política al responsabilizarla, en parte, de las altas tasas de mortalidad infantil que asolaban la infancia. Dicha percepción quedó plasmada en la Ley del 12 de agosto de $1904^{10}$, cuyo artículo tercero estableció un Consejo Superior de Protección a la Infancia, con funciones como las siguientes:

- Vigilar periódicamente a los niños sometidos a lactancia mercenaria, procedieran de inclusas o de sus propios hogares,

- Controlar a las nodrizas a través de documentos,

- Promover la salud de éstas y los pagos por su trabajo,

- Recompensar a aquellas nodrizas que lo mereciesen,

- Observar las disposiciones sanitarias y el orden interior de los establecimientos dedicados a la recogida de niños ${ }^{11}$.

\section{REGULACIÓN DE LA LACTANCIA EN LA INCLUSA DE TOLEDO A PRINCIPIOS DEL SIGLO XX}

La realidad de la inclusa toledana es similar a la de otras casas cuna de la geografía española, aunque se observa a comienzos del siglo XX un endurecimiento de la normativa respecto a los requisitos exigidos a quienes solicitaban ser nodrizas. Con ello no solo se perseguía evitar los probables abusos de años precedentes, mas también reforzar el control social al restringir deliberadamente el acceso a la remuneración económica por lactancia a las mujeres más estigmatizadas socialmente como madres solteras, prostitutas y viudas con hijos de relaciones ilegítimas ${ }^{12}$. Una herramienta de poder cultural, pues, muy potente.

Cualquier niño que llegaba a la inclusa, independientemente de la modalidad utilizada para su abandono (torno, Casa de Maternidad o abandono callejero), era registrado y si era menor de dieciocho meses quedaba consignado a la sección de lactancia, donde, en principio, era amamantado por las amas de cría del establecimiento ${ }^{13}$. Según el propio reglamento contemplaba, su número no podía ser menor al necesario, basándose esta necesidad en la media de ingresos del quinquenio anterior.

También se reconocía el compromiso de la Diputación a la hora de garantizarles su estipendio y alimentación, a cambio, claro está, del correcto desempeño 
de su tarea ${ }^{14}$. Antes de su ingreso, las nodrizas del establecimiento toledano eran reconocidas por el director médico, quien volvía a verificar su estado de salud cada quince días. Quedaban al cuidado de una religiosa, hermana de la Caridad, encargada de distribuir a los lactantes de forma equitativa, así como de vigilar el desempeño correcto de su trabajo y hacer cumplir las estrictas normas de conducta que regían el establecimiento ${ }^{15}$.

La frecuente carencia de nodrizas internas hacía necesario buscar fuera de la institución alternativas para los recién nacidos, a quienes se procuraba integrar, de este modo, en un medio familiar encargado de su alimentación y cuidado general ${ }^{16}$. La Diputación de Toledo, en el artículo 28 del Reglamento de los Establecimientos Reunidos, establecía los requisitos exigidos a las mujeres que quisiesen llevarse un niño de la inclusa para amamantarlo ${ }^{17}$ :

«Las mujeres que soliciten la entrega de algún expósito para la lactancia, acudirán con solicitud al director, en la cual se haga constar la licencia de su marido, informada por el alcalde, cura párroco, juez municipal y médico titular, respecto a la edad, conducta moral y religiosa de los cónyuges, si padecen o han padecido alguna enfermedad de carácter crónico o infeccioso, nombres y apellidos, profesión y señas del domicilio del marido.

Acreditar que la leche no tiene más tiempo que el de seis meses.

Que el niño que estaba criando la solicitante ha fa\|lecido» ${ }^{18}$.

\section{El artículo 29 recoge que:}

«La dirección podrá solicitar informes públicos o reservados, y con vista de todo y del reconocimiento facultativo sobre la calidad y cantidad de la leche, entregará o no al expósito» ${ }^{19}$.

Con el artículo 30 se evitaba que las solicitantes eligieran el expósito que se llevaban y que este procediera de su mismo lugar de residencia. Finalmente, también podemos subrayar que el artículo 31 prohibía «Entregar ningún niño expósito, tanto de lactancia como de destete, a mujeres solteras, aunque renuncien el estipendio ${ }^{20}$.

Como puede verse, se deduce por la normativa la preocupación que los gestores tenían por minimizar los posibles abusos que se daban en torno al nodrizaje y reforzaron, en esta dirección, las exigencias requeridas para ser ama externa ${ }^{21}$. También se pedía que el niño de la solicitante hubiese fallecido, en un intento por evitar que el negocio de la lactancia fuese un estímulo para el abandono de los hijos legítimos.
Sin embargo, había criterios que se obviaban ante la carencia de amas. Sabemos de madres que ya habían lactado a vecinos suyos antes de acudir a la institución o bien a sus propios hijos, aunque el Reglamento indicaba que la leche no debía tener más de seis meses. El reconocimiento médico a la nodriza trataba de impedir la propagación de enfermedades infecciosas como la sífilis, aunque raramente eran rechazadas por la calidad de su secreción.

\section{MORTALIDAD DE LOS EXPÓSITOS AMAMANTADOS POR NODRIZAS}

El análisis de la mortalidad expósita ha sido objeto historiográfico en el campo de la infancia abandonada, puesto que se mantuvieron tasas muy elevadas de fallecimientos entre los alojados en este tipo de instituciones, independientemente del lugar y la época de estudio. En nuestro caso el interés deriva de poder analizar una cohorte de niños que nacieron en la inclusa toledana bajo circunstancias homogéneas y, por ello, teóricamente resguardados de las peores condiciones que se daban en los nacimientos clandestinos y posteriores traslados a la inclusa. Con ese grupo podemos calcular tasas fiables sobre diversos perfiles de su mortalidad: diferencias entre las defunciones producidas en los niños que salían a lactar con nodrizas externas y los que quedaban en la inclusa, tasas de mortalidad por grupos de edad en ambos entornos, etiología de los óbitos, comparación con lo que ocurría con otras inclusas españolas y con la mortalidad general de la población infantil. A partir de estos detalles podremos mostrar un panorama global de la mortalidad expósita en el centro benéfico de Toledo y compararlo con otros casos de la geografía nacional ya conocidos.

Para estimar la tasa de mortalidad general en este grupo concreto de niños se ha utilizado el cociente entre las defunciones en el período de estudio y el total de niños nacidos en la Maternidad abandonados en la institución. Se han excluido deliberadamente del cómputo las muertes perinatales (las producidas al nacer o en las primeras veinticuatro horas de vida), porque muchos de ellos no se pueden contabilizar como nacidos vivos al ser abortos o fetos muertos y porque en el resto, la influencia de los cuidados prestados o del funcionamiento institucional era escasa, pues en gran medida el deceso estaba causado por problemas asociados al embarazo o al parto. Esta tasa es del 549 por mil en el grupo nacido entre 1900 y 1930. De los 857 niños nacidos que cumplieron las 24 horas de vida, fallecieron 471 durante los primeros cinco años, período de vida del expósito en el que se conoce con certeza lo que le 
sucedió (desde esa edad cesaba el cuidado remunerado y la institución no registraba de una manera sistematizada las defunciones). Las cifras están en consonancia con las que presentaban otras inclusas españolas en los primeros años del siglo XX, pero la tasa es inferior a la que calcula B. Revuelta en la inclusa de Madrid ${ }^{22}$.

Para ser más precisos en el estudio de la mortalidad utilizamos la tasa de mortalidad infantil, es decir, el cociente entre los fallecimientos producidos durante el primer año de vida (excluidas las muertes perinatales) y el número de nacidos en la Maternidad expuestos a morir en el período analizado. En la cohorte estudiada es un indicador que aporta información muy precisa, ya que la mayor parte de las muertes se producían durante ese primer año, al ser un período vital de máxima vulnerabilidad. En la institución que analizamos fue del $396,7 \% 0^{23}$, cifra muy superior ${ }^{24}$ a la que tenía la provincia de Toledo en el quinquenio 1901-1905 (192,39 \%o según Gómez Redondo, 1992, p. 45) y alejada también de las pautas de la mortalidad infantil en España durante la primera década del siglo $X X$, ya que estas cifras muestran un descenso mantenido a medida que avanzan los años, desde 185,9 \%o en 1901 hasta el 145,3 \%。 en 1910 (Gómez Redondo, 1992, p. 34) ${ }^{25}$.

Las tasas generales de mortalidad por grupo de edad se han hallado como el cociente del número de fallecidos en ese grupo de edad entre el total de expuestos a morir en ese mismo grupo, es decir, se han excluido las defunciones y los niños que fueron recuperados en el tramo anterior de edad, para no subestimar la mortalidad al poner en el denominador sujetos que no estaban en riesgo de morir en la inclusa (gráfico 1). Se ha desglosado el número de fallecimientos de la cohorte por tramos de edad, estableciendo varias categorías frecuentemente usadas en los análisis sobre mortalidad infantil:

- Neonatal: número de niños fallecidos durante los primeros veintisiete días de vida extrauterina, excluyendo los que murieron en las primeras 24 horas. Aunque no se pueden descartar que algunas estén causadas por enfermedades congénitas o por problemas asociados al embarazo y al parto, en ellas tiene influencia los cuidados sanitarios que se prestan al nacer y la disponibilidad de alimentos. Esta tasa es del 72,3\%.

- Posneonatal: número de niños fallecidos entre los 28 y 365 días de vida extrauterina. En este periodo, las muertes están muy relacionadas con causas ambientales y socioeconómicas, como la falta de salubridad e higiene, la alimentación deficiente, el hacinamiento... En el caso que se estudia, tendrían una relación directa con los cuidados que recibía el menor en la institución o en las familias de acogida y estaría estrechamente vinculado a la alimentación, pues es la fase en la que la lactancia materna era el alimento básico que recibían estos niños. Esta tasa es del 361,9\%.

Gráfico 1. Tasas de mortalidad por grupos de edad. Expósitos nacidos en la Maternidad, 1900-1930

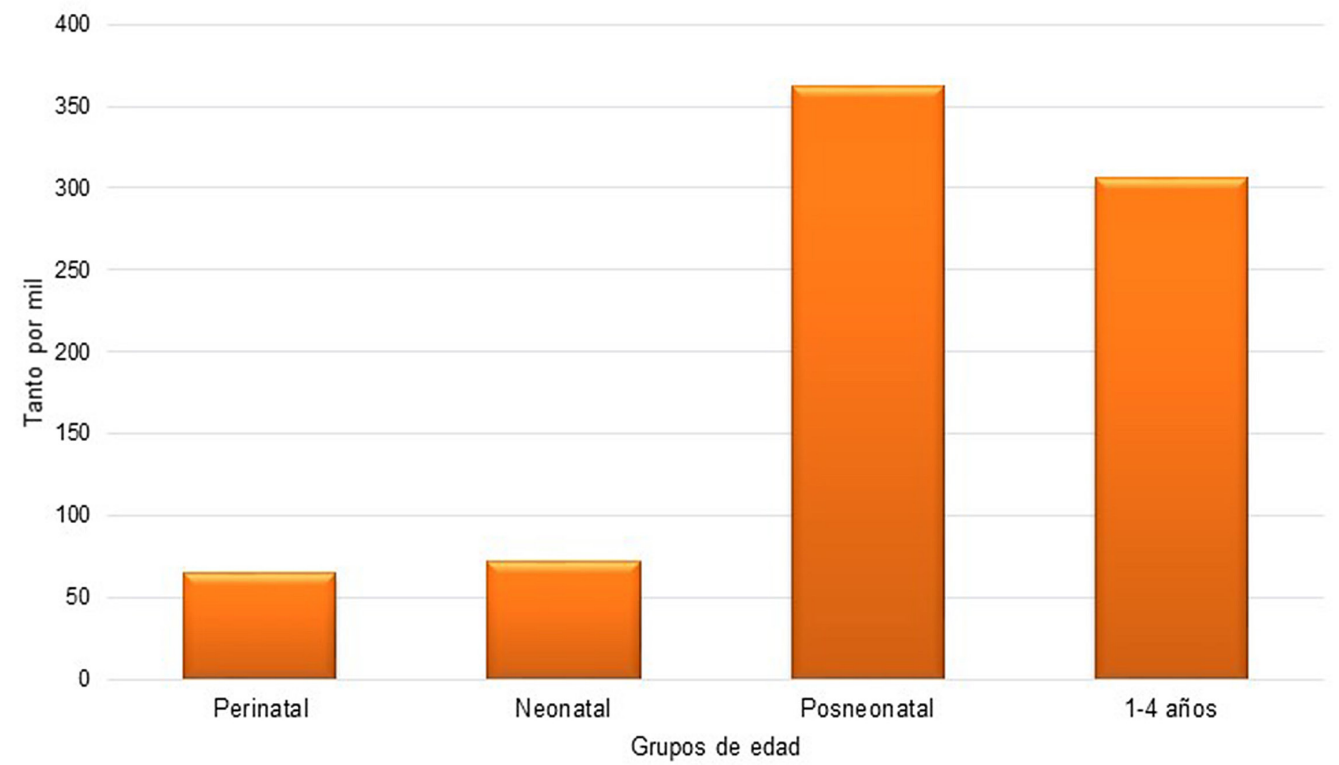

Las tasas de mortalidad se han hallado poniendo en el numerador las defunciones en cada grupo de edad y en el denominador la población expuesta a morir en ese grupo. Fuente: Libros de lactancias y destetes. 1900-1930. A.D.P.T. Elaboración propia 
- Juvenil: entre el primer y el quinto aniversario. Este intervalo se considera un indicador de las condiciones de vida de las poblaciones, ya que los niños habían abandonado la lactancia materna y estaban sujetos a los condicionantes socioeconómicos del ambiente en el que se criaban, por lo que es muy sensible a las variaciones producidas en ellas. Esta tasa es del 306,07 \% para la cohorte analizada.

La distribución de la mortalidad por edades muestra que la más elevada se daba en el período posneonatal, antes de cumplir el año de vida. La probabilidad de morir disminuía ligeramente a medida que aumentaba la edad del expósito, aunque la tasa de mortalidad juvenil se mantenía muy elevada. Si lo comparamos con las tasas referidas a la población española, vemos que la mortalidad infantil de la inclusa duplicaba a la de la población general, estimada en 180,2 \% en 1906 y 119,6 \%。 en 1932, manteniéndose esa misma elevación respecto a la mortalidad juvenil poblacional que era de $172,6 \%$ en 1906 y de 75,6 \%o en 1932 (Sanz, 2001, p. 137). En este primer tercio del siglo XX, los valores de la mortalidad juvenil eran inferiores a los de la mortalidad infantil, lo que parece indicar que España estaba en un proceso de transición demográfica equiparable al de los países del norte de Europa, que seguían esta pauta desde finales del siglo XIX. En la institución que analizamos se da la misma relación entre mortalidad infantil y juvenil que en la población general, puesto que la juvenil se mantiene en niveles inferiores y es similar a lo que ocurrió en otras inclusas españolas en el período de estudio ${ }^{26}$.

Debemos mantener cautela en la explicación de estas tasas, puesto que una elevada mortalidad juvenil se puede atribuir a unas deficientes condiciones de alimentación, salubridad y cuidado de las familias de acogida, explicación plausible si tenemos en cuenta que los padres nutrices eran jornaleros pobres que acogían al expósito para completar sus ganancias mensuales y, posiblemente, no tenían acceso a una alimentación adecuada ni contaban en sus hogares con unas condiciones higiénico-sanitarias mínimas. Pero tampoco debemos olvidar que cada expósito tenía una experiencia previa, dentro y fuera de la institución, y esta podía condicionar su supervivencia futura ${ }^{27}$. Otros autores atribuyen un efecto scarring, de debilitamiento o daño permanente, a la estancia de los pequeños en el ambiente insalubre de la inclusa, que condicionaría su salud futura (Revuelta, 2011, p. 406). De cualquier modo, el acogido estaba expuesto a todas las condiciones ambientales y económicas desfavorables que pudieran afectar a su familia nutriz, pero eso no es indicativo de que se le tratase negligentemente de manera intencionada.
Para tener una panorámica de las diferencias que había en la mortalidad entre los niños que nunca abandonaron la Maternidad y los que salieron a lactar con nodrizas externas, es necesario recurrir al análisis de mortalidad por grupos de edad y lugar de lactancia. Esta aproximación tiene serias limitaciones, puesto que no eran dos grupos homogéneos y un sesgo de selección determinaba qué expósitos salían a lactar y cuáles se quedaban en la institución. Los nacidos con patologías congénitas o con bajo peso eran amamantados por nodrizas internas y morían en pocos días. Aquellos que superaban el mes de vida y permanecían en el centro benéfico sufrían el ambiente patológico de la inclusa, con nodrizas compartidas por varios niños, convirtiéndose en vector de transmisión de enfermedades y expuestos al hacinamiento ocasionado por los nuevos ingresos que se sumaban a los retrasos en la incorporación a las familias de acogida ${ }^{28}$. Por su parte, la mayoría de supervivientes que no salieron de la institución fueron reconocidos por sus familias biológicas en los primeros días o meses de vida, lo que les permitió sobrevivir.

Para realizar este análisis se han considerado dos grupos de niños: los que no salieron nunca a lactar fuera de la institución y aquellos que fueron acogidos por nodrizas externas al centro benéfico. Hemos excluido del análisis a 71 niños que salieron a lactar, pero finalmente fueron devueltos a la institución y fallecieron en ella, debido a que no disponemos de información con la que determinar el tiempo de permanencia en el centro benéfico antes de fallecer, ni podemos precisar si las causas del fallecimiento estaban relacionadas con la experiencia en la familia de acogida, con condicionamientos intrínsecos o con la influencia del periodo de institucionalización. Además, también descartamos los 6 casos de los que no se han conservado datos. Hechas las precisiones metodológicas, podemos afirmar que la tasa de mortalidad bruta de los niños que no salieron a lactar fue del 647,05 \%o y la de los que sí salieron del 458,62 \%o. Estas diferencias posiblemente se deban al sesgo de selección anteriormente descrito, que indudablemente aumentaba la mortalidad en la inclusa. Es difícil calibrar qué influencia tuvo el trabajo de las nodrizas internas en estas tasas de mortalidad tan elevadas, pues resulta imposible desagregar la contribución de otras causas relevantes como patología presente al nacer, bajo peso o debilidad congénita. Aun suponiendo que amamantasen adecuadamente a los niños, hay factores que podían influir negativamente en la lactancia, como un estado de salud deficiente o la calidad de la leche. Incluso, no podemos dejar de insistir en el número insuficiente de nodrizas que la institución tuvo constantemente. 
Las tasas por grupo de edad y lugar de lactancia se han hallado poniendo en el numerador los niños fallecidos en ese grupo de edad en un lugar determinado y en el denominador los susceptibles de morir en ese lugar y grupo de edad (gráfico 2).

Observamos que la mortalidad neonatal ocurre en la casa-cuna, debido a que los niños que nacían debilitados o con patologías congénitas no salían a lactar y morían los primeros días de vida. La mortalidad posneonatal era más elevada en la inclusa que en las familias de acogida, porque los niños con problemas de salud tampoco salían a lactar y si no se lograba alojarlos con nodrizas externas en un corto periodo de tiempo, el propio ambiente patológico de la institución -en el que los lactantes compartían las amas y espaciospropiciaba la transmisión de enfermedades. La mortalidad juvenil ocurría exclusivamente con las familias de acogida, porque de los 204 niños que no abandonaron la inclusa, tan solo 9 tenían entre 1 y 4 años, y fueron reconocidos en ese tramo de edad. Esta tasa se mantiene muy elevada, pero es algo inferior a la de mortalidad posneonatal para los acogidos por familias, probablemente porque los niños que habían sobrevivido eran los más fuertes y ya no necesitaban exclusivamente la lactancia materna para su alimentación, por lo que no dependían de que la nodriza tuviese una cantidad y calidad adecuada de secreción láctea, aun cuando siguieran expuestos a las condiciones materiales e higiénico-sanitarias que les rodeaban.

Podemos completar el análisis refiriéndonos a los diagnósticos sobre la causalidad de la muerte que permitirían la comparación con el conjunto de la población española en esos tramos de edad. Es una tarea compleja en la medida que está influida por factores de muy difícil control como la aplicación de criterios diagnósticos distintos (médicos o populares), los cambios en la nomenclatura y clasificación de muerte del momento, o la ocultación de enfermedades por estigmatización social $^{29}$. En este sentido, algunos autores han señalado la problemática del diagnóstico retrospectivo y han formulado nuevas tablas de expresiones diagnósticas que aglutinen las que se utilizaban y permitan la comparabilidad de los resultados (Bernabeu, Ramiro, Sanz y Robles, 2003). En nuestro caso, hemos procedido a una clasificación reducida de esta propuesta que nos permita la comparabilidad de resultados (Tabla 1).

A nivel general, es posible relacionar los tramos de edad de los fallecimientos con las patologías consignadas como causas de las defunciones, para comprobar en qué medida a cada grupo de edad le afectaban unas enfermedades asociadas a la herencia y al desarrollo del embarazo u otras, más ligadas a los contextos socioeconómicos en los que se desarrollaba la crianza de los expósitos ${ }^{30}$ (gráfico 3 ).

Gráfico 2. Tasas de mortalidad por grupos de edad y lugar de lactancia. Expósitos nacidos en la Maternidad, 1900-1930

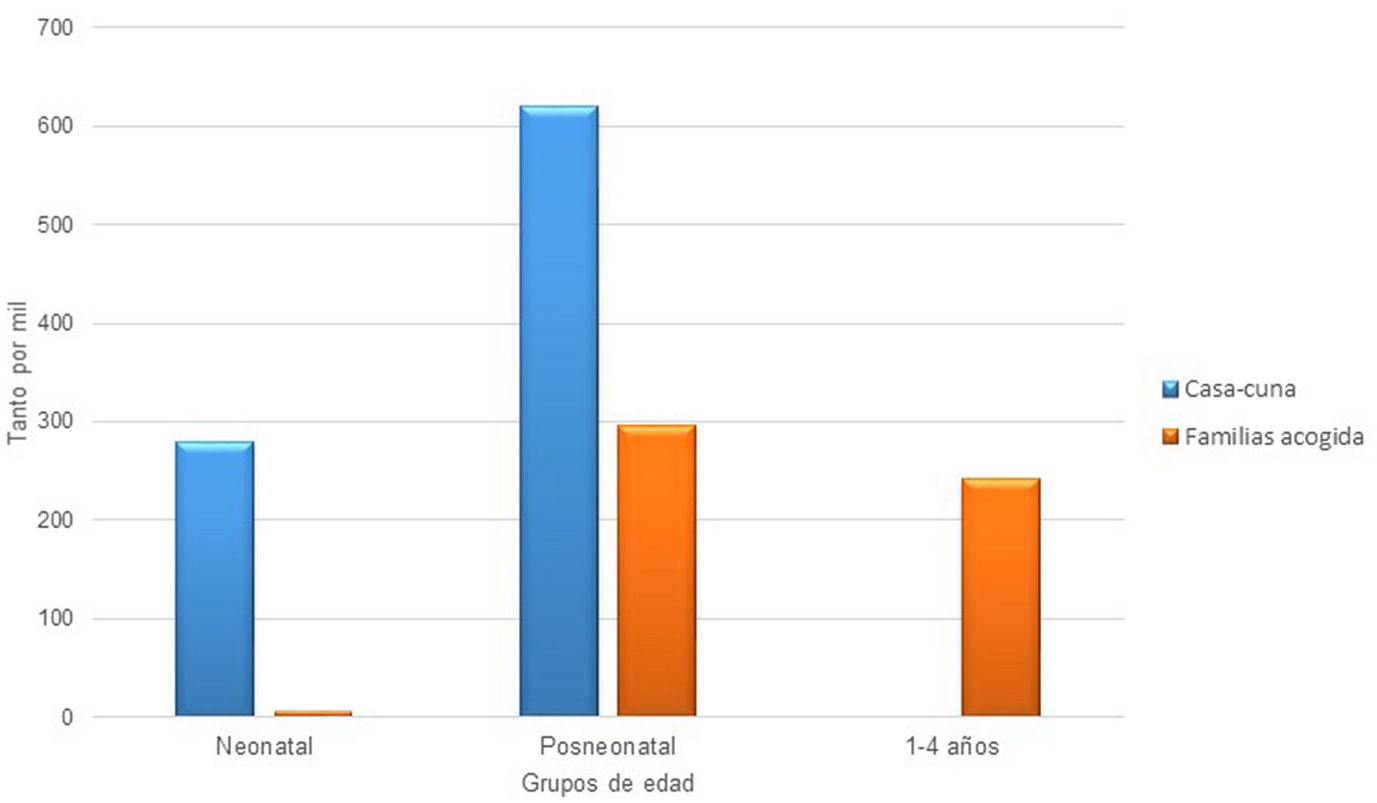

Las tasas de mortalidad se han hallado poniendo en el numerador las defunciones en cada grupo de edad y lugar de lactancia y en el denominador la población expuesta a morir en ese grupo y lugar. Fuente: Libros de lactancias y destetes. 1900-1930. A.D.P.T. Elaboración propia 
Tabla 1. Clasificación de diagnósticos simplificada

\begin{tabular}{|c|}
\hline CLASIFICACIÓN DE DIAGNÓSTICOS \\
\hline Atrepsia \\
\hline Eclampsia \\
\hline Infecciones respiratorias \\
\hline Infecciones digestivas \\
\hline Otras infecciones \\
\hline Enfermedad no infecciosa \\
\hline Falta de desarrollo/raquitismo \\
\hline Sífilis \\
\hline Causa desconocida \\
\hline
\end{tabular}

Fuente: simplificación de la clasificación propuesta por Bernabeu et al. (2003). "El análisis histórico de la mortalidad por causas. Problemas y soluciones". Revista de Demografía Histórica, 21 (1), pp. 176-183. Elaboración propia.
Las tasas de mortalidad por causas y grupos de edad se han hallado poniendo en el numerador las defunciones por esa causa en ese grupo de edad y en el denominador los niños expuestos a morir en ese grupo.

En la mortalidad neonatal vemos que la patología que prevalece sobre las demás es el raquitismo (a veces denominada falta de desarrollo físico), seguida de la atrepsia y la eclampsia, cuyo origen es congénito si está diagnosticada con precisión. Se evidencia, pues, que hasta el día 28 de vida seguían teniendo mucha influencia las dolencias hereditarias o las asociadas a complicaciones gestacionales, es decir, aquellas con un fuerte componente endógeno ${ }^{31}$. El raquitismo se asociaba a partos prematuros o a niños que nacían con bajo peso, lo que resultaba un factor determinante para su grado de supervivencia. Aparecen en menor medida las infecciones y otras de etiología no infecciosa, lo que evidencia que el entorno no tenía aún una influencia determinante en la explicación de parte de la mortalidad que afectaba a este grupo de edad.

En el periodo posneonatal destacan las infecciones como causa principal de la mortalidad, seguida de la atrepsia, que a veces enmascaraba trastornos digestivos relacionados con procesos infecciosos ${ }^{32}$. La patología denominada raquitismo o falta de desarrollo físico permanece estable respecto al grupo de edad anterior, lo que indicaría que hasta el año de vida la

Gráfico 3. Tasas de mortalidad por causas según grupos de edad. Expósitos nacidos en la Maternidad, 1900-1930

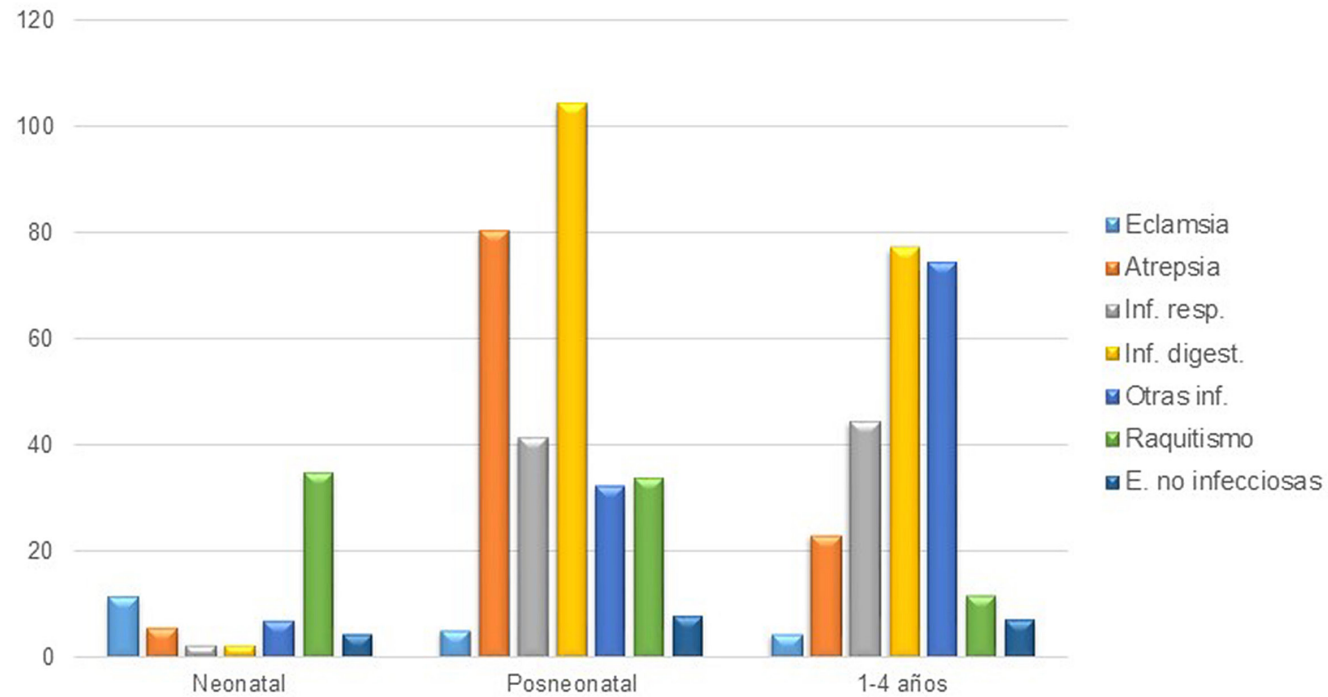

Las tasas de mortalidad por causas se han hallado poniendo en el numerador las defunciones por cada causa en cada grupo de edad y en el denominador la población expuesta a morir en ese grupo de edad. Un $15,5 \%$ de las defunciones no consignaba la causa de muerte.

Fuente: Libros de lactancias y destetes. 1900-1930. A.D.P.T. Elaboración propia. 
lactancia tenía un papel determinante en la supervivencia de los pequeños, muy sensibles a la escasez de amas, a la calidad y calidad de la leche suministrada y a la ratio niños/nodriza por inclusa.

En el grupo de 1-4 años la mayor parte de la mortalidad es atribuible a patologías infecciosas, algo indicativo de las deficientes condiciones higiénicos-sanitarias del entorno en el que se criaban los expósitos; en este caso, todos ellos con familias rurales. Disminuyen considerablemente las muertes atribuidas al raquitismo o a una alimentación deficiente. Es decir, se alimentaba mejor a los expósitos, pero quedaban expuestos a otros riesgos ambientales igualmente letales como la contaminación de alimentos perecederos por el calor, la carencia de agua potable y de una red de alcantarillado, el hacinamiento o la convivencia con animales domésticos; factores todos ellos que propiciaban la diseminación de enfermedades infecciosas.

Para tener una visión más completa de lo que sucedió con los niños fallecidos en la institución, dependientes de los cuidados de las nodrizas internas, y aquellos que lo hicieron con amas externas, hemos analizado la mortalidad y sus causas atendiendo a la pertenencia a uno u otro grupo. En este sentido nos hemos fijado en la mortalidad por causas en el perío- do posneonatal, ya que en esta etapa se producía la mayor parte de los fallecimientos, el número de decesos es similar y, además, se trata del período infantil en el que la alimentación materna y los cuidados recibidos por el lactante son determinantes para su supervivencia (gráfico 4). La tasa representada en el gráfico resulta del cociente entre el número de defunciones por una causa determinada en ese lugar, en el período posneonatal, y los niños en riesgo de morir en ese mismo período y lugar.

Entre los fallecidos en la institución, las causas de muerte que más aparecen son la atrepsia, el raquitismo y las infecciones digestivas. El raquitismo podía deberse a problemas congénitos como el bajo peso, a veces originado por embarazos poco saludables, en los que las cargas de trabajo, la escasa alimentación y la falta de cuidados apropiados originaban partos prematuros o niños nacidos con un peso insuficiente. En otros casos, sería posible relacionarlo con la deficiente alimentación que recibían los pequeños en la inclusa y que les conducía a unas situaciones de desnutrición tan severa como para provocarles la muerte.

La atrepsia es una enfermedad propia de los recién nacidos y niños de corta edad que se caracteriza por la desnutrición lenta y progresiva a consecuencia de

Gráfico 4. Tasas de mortalidad por causas según lugar de lactancia. Periodo posneonatal. Expósitos nacidos en la Maternidad, 1900-1930

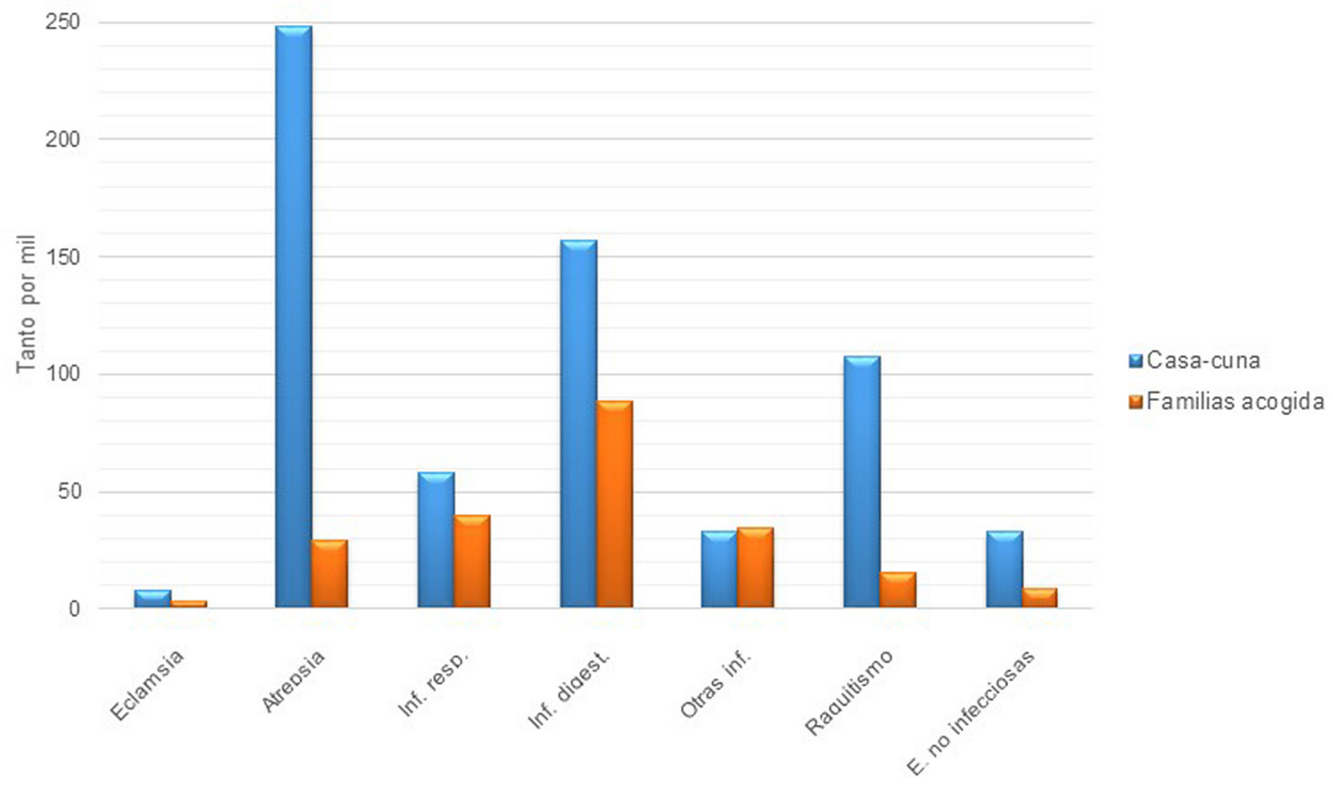

Las tasas de mortalidad por causas según lugar de lactancia se han hallado poniendo en el numerador las defunciones por cada causa en el periodo posneonatal en ese lugar de lactancia y en el denominador la población expuesta a morir en ese determinado lugar y grupo de edad.

Fuente: Libros de lactancias y destetes. 1900-1930. A.D.P.T. Elaboración propia 
la imposibilidad de digerir los alimentos (Sanz y Ramiro, 2002). Puede deberse a múltiples causas como una alimentación insuficiente, procesos digestivos agudos o crónicos, infecciones, defectos o anomalías del aparato digestivo... Es una patología que presenta en las fuentes manejadas un fuerte componente estacional, concentrándose en los meses de más calor, entre mayo y agosto un $56,4 \%$ de los casos etiquetados como tal. Ello sugiere que posiblemente la expresión enmascarase infecciones digestivas diversas, que cursaban con una sintomatología similar, confundiendo ambos diagnósticos, ya que es improbable que la estacionalidad afecte a una patología congénita $^{33}$. Habitualmente se desplaza esta mortalidad a las infecciones digestivas, pues era frecuente intercambiar el diagnóstico de enteritis con el de atrepsia ${ }^{34}$. Si tenemos en cuenta esta suposición, en los niños que no salieron de la inclusa, la tasa de mortalidad relacionada con procesos digestivos, de origen infeccioso o no, estaría en torno al 40 \%, lo que apuntaría, más que a la deficiente alimentación, a la precaria higiene del establecimiento como responsable determinante en la explicación de la mortalidad. En ese escenario caben fómites infectados, utensilios en general manipulados incorrectamente y nodrizas compartidas sin asepsia ${ }^{35}$. En total, aproximadamente un $50 \%$ del grupo de niños que permaneció en la inclusa moría de patología infecciosa en el período de estudio, cifra bastante coincidente con la que aporta B. Revuelta para la inclusa de Madrid en el mismo grupo de edad y periodo (Revuelta, 2011).

En el caso de los expósitos criados por amas externas, la atrepsia y el raquitismo tienen una representación limitada, mientras que a las infecciones digestivas, respiratorias y otras sin definir se les atribuyen la mayor parte de los fallecimientos. Si volcamos los datos de la atrepsia en el capítulo de enfermedades infecciosas digestivas - pues al igual que en el caso anterior el $40 \%$ de los diagnósticos de atrepsia ocurrían en los meses de más calor - tendríamos que el $12 \%$ de los niños que salieron a lactar con nodrizas externas, murieron por esta causa. En estos niños, la etiología principal por la que fallecían tenía un origen infeccioso en el $19 \%$ de los acogidos, frente a un $3 \%$ debida a etiología no infecciosa. Esto puede ser indicativo de que la lactancia se realizaba generalmente de modo correcto, aunque existían importantes deficiencias en materia de sanidad e higiene públicas y en los cuidados referidos a la alimentación infantil, pues se desconocían las normas de manipulación de alimentos que hoy consideramos elemen- tales y las prácticas higiénicas eran, por lo general, bastante precarias en aquel entonces. Es interesante comparar estas tasas con lo que ocurría con la población infantil española en ese grupo de edad. Sanz Gimeno estima en 1906 una tasa de mortalidad por enfermedades digestivas del 54,2 \%o, por enfermedades respiratorias del 35,5\% y, finalmente, por enfermedades congénitas del 26,6 \%. Para 1932, 41,2 \%o por enfermedades digestivas, $27,7 \%$ respiratorias y $26,2 \%$ congénitas. En los niños lactados con familias de acogida estas tasas alcanzaron para la patología digestiva el 88,5 \% (118\%o si incluimos la atrepsia), la respiratoria llega al $40 \%$ y la congénita se queda en el $15 \%{ }^{36}$. Son índices ligeramente superiores a los de la población general, pero mantienen la proporcionalidad en cuanto a la representación etiológica. Este paralelismo se explica porque los niños lactados vivían en entornos similares a los del resto de población rural y estaban, pues, afectados por los mismos factores. Quizás la elevación de tasas se deba a que las familias que criaban expósitos procedían de los grupos más empobrecidos, en los que el cabeza de familia era jornalero o pastor, y, por lo tanto, sus condiciones económicas de supervivencia eran inferiores a la media poblacional.

Para comprobar con mayor precisión esta asimilación entre las causas de mortalidad de la población expósita que lactaba en zonas rurales y las de la población infantil general en la fecha de estudio, hemos estimado la contribución porcentual de las patologías más relevantes respecto al total de fallecimientos en niños de 0-5 años criados por familias nutrices. El resultado obtenido es que las infecciones digestivas causaban el $16,8 \%$ de los fallecimientos, la atrepsia el $4 \%$ y las infecciones respiratorias el 6,6\%. Si además unimos la atrepsia a la patología digestiva nos encontramos con un $20,8 \%$ de los fallecimientos. Comparados los datos con los porcentajes que ofrece Sanz Gimeno para la provincia de Toledo en la población infantil general, observamos coincidencias: las enfermedades del aparato digestivo en 1906 representaban entre el 24 y el $33 \%$ del total de causas de muerte y entre el 34 y el $42 \%$ en 1932. Las enfermedades del aparato respiratorio representaban del 11 al $18 \%$ en 1906 y del 17 al $24 \%$ en 1932. Esta comparación refuerza la tesis de que los expósitos lactados en zonas rurales tenían causas de mortalidad semejantes al resto de la población infantil, ya que eran las condiciones de vida de las clases populares las que las determinaban en gran medida y no el cuidado negligente de las familias que los cuidaban. 


\section{EPÍLOGO}

El estudio de los niños abandonados y nacidos en la Casa de Maternidad de Toledo pone de manifiesto que sus altas tasas de mortalidad no tenían una explicación unívoca, sino que se debían a varios factores imbricados. En el caso de los fallecimientos durante las primeras horas o días de vida se deben relacionar con la gestación y si hablamos de una muerte más tardía, habrá que rastrear al expósito desde la inclusa hasta la lactancia externa en zonas rurales. Allí quedaba expuesto a las condiciones de su familia nutriz, generalmente pobre y que recurría a la lactancia asalariada para completar sus ingresos básicos. Como hemos visto, las tasas de mortalidad de los expósitos

\section{NOTAS}

1. Varias investigaciones europeas sobre niños ilegítimos señalan que las tasas de mortalidad de estos eran superiores a la de los legítimos y que en ocasiones la quintuplicaban. Alojar al niño en una institución no significaba su supervivencia, pues la mortalidad superaba el $500 \%$ (Luddy, 2001). En Escocia se constata este mismo hecho, las tasas de mortalidad infantil eran más altas en las zonas urbanas escocesas, pero la situación se invertía cuando se trataba de ilegítimos, que morían con más frecuencia en las zonas rurales (Blaikie, 1998).

2. La mortalidad en las inclusas españolas en el siglo XVIII ha sido estudiada en varias zonas geográficas: Almería (Gómez, 1997); Granada (de la Fuente, 2000); Salamanca (Fernández, 1988); Sevilla (Álvarez, 1980); Úbeda (Tarifa, 1994). Varios trabajos recogen el mismo tema para el siglo XIX: Guipúzcoa y Navarra (Valverde, 1994); Madrid (Vidal, 1995); Salamanca (Torrubia, 2004); Zamora (Galicia, 1985).

3. Los puericultores españoles de principios del siglo XX defendieron la lactancia materna frente a la artificial, que solo justificaron en casos de padecimiento de sífilis o tuberculosis o por inexistencia de secreción láctea. El caso de Palma ha sido documentado por Joana Pujades (Pujades, 2008). En España (desde 1902) y en Europa, las Gotas de Leche vigilaban la lactancia materna, decidían qué niños precisaban de lactancia mixta y se encargaban del reparto diario gratuito o semigratuito de leche esterilizada, cuando la lactancia materna era inviable (Rodríguez Ocaña, 1985; 2001).

4. B. Revuelta documenta en su tesis que la lactancia artificial y la mixta eran una realidad en la mayor parte de las inclusas nacionales, aunque al principio tuvo trágicos resultados en Zaragoza, Huesca o Lérida, y fue mejorando poco a poco en los años posteriores, cuando se establecían controles como las Gotas de Leche, como ocurrió en Navarra (Revuelta, 2012).

5. Concepción Arenal las definía como "mujeres mal alimentadas que dan al expósito el alimento necesario para que arrastre lánguidamente una vida que no tarda en extin- criados con nodrizas externas eran superiores a las de la población rural general, posiblemente por la influencia del efecto scarring institucional y por la dura experiencia vital acumulada. Si existen diferencias en las tasas de ambos grupos, las causas de la mortalidad son similares; es decir, el papel fundamental en el mantenimiento de una mortalidad elevada corresponde a las penosas condiciones de vida, tanto de la población infantil expósita como de la que no lo era. Aun cuando carezcamos de información sobre el modo en el que las amas externas desarrollaban su trabajo, difícilmente podríamos sostener la imagen de un nodrizaje negligente cuando más de la mitad de los lactantes (el $54 \%$ ) conseguía sobrevivir.

guirse" (Arenal, 1861; 35). En el mismo sentido, un artículo de José Ortega Munilla titulado "La nodriza-ogro" (Ortega, $1883 ; 140-141)$.

6. El impacto de la práctica del nodrizaje en la mortalidad infantil se ha abordado en varios trabajos (Bardet, Dufour y Renard, 1997; Sussman, 1975). Otro tema tratado por la historiografía ha sido el de la imagen social de la nodriza (Morel, 2010).

7. En esta línea el artículo de Testón y otros sobre el trabajo de las amas de cría en Puebla de Guadalupe en los siglos XVIII y XIX (Testón, Sánchez y Blanco, 2004).

8. Comunicación del año 1925 que se encuentra en el legajo 180. A.D.P.T.

9. El perfil social de las nodrizas internas y externas de la inclusa de Toledo en la tesis doctoral inédita de Martín Espinosa (2012).

10. La importancia de esta ley para la protección de la infancia se ha recogido en varios estudios (Barona, 2004; Perdiguero y Robles, 2004)

11. Para conocer los artículos de la Ley de 12 de agosto de 1904, véase Gaceta de Madrid, núm. 230 (17-8-1904).

12. Para todas las nodrizas, la ley de 12 de agosto de 1904 determinaba en su artículo octavo que las mujeres que quisieran ejercer de nodrizas tenían que presentar ante la Junta local un documento que especificase: su estado civil, salud, conducta y condiciones físicas, el permiso del marido si era casada y la partida de nacimiento del hijo para acreditar que éste tenía entre seis y diez meses o certificado de que estaba siendo criado por otra mujer.

13. Solo se tienen datos del número de nodrizas internas que había en la Casa-Cuna toledana en el período 1900-1910. En esas fechas fueron abandonados una media 90 niños por año, y hubo un promedio de 9 nodrizas anuales, aunque estas cifras hay que tomarlas con cautela, puesto que gran 
parte de los niños lactaban en la institución unos días antes de ser alojados con nodrizas externas, por lo que nunca permanecían a la vez el número total de ingresados. A modo de ejemplo, en 1900 se abandonaron 109 niños y trabajaron en la institución 15 nodrizas, aunque no todas estuvieron el año completo. Libro nóminas amas internas (1903-1910). A.D.P.T.

14. Normativa recogida en el capítulo III del título II del Reglamento de los Establecimientos Reunidos de Toledo, Toledo, 1902 , p. 7.

15. En las inclusas italianas se refleja una situación similar, ya que las nodrizas internas siempre eran escasas debido a las difíciles condiciones de vida de las inclusas, pues tenían que alimentar a varios niños a la vez, los salarios eran bajos y les obligaban a vivir en la institución, alejadas de sus familias. Esta imposibilidad para reclutar amas internas hizo que se recurriese a las madres solteras que habían dado a luz en la maternidad y habían dejado a su hijo en la inclusa, aunque no se les permitía lactar a su hijo para evitar que su preferencia hacia él fomentase la mala alimentación de los otros. La vida de estas mujeres como empleadas de la inclusa seguía un régimen carcelario: sólo hablaban con su familia una vez a la semana, podían salir del centro en grupos y escoltadas y no se les permitía hablar con extraños (Kertzer, 1991).

16. No era la única razón, ya que las instituciones sabían que las posibilidades de supervivencia para los niños aumentaban si se les alojaba cuanto antes con una nodriza externa, puesto que las épocas en las que estas escaseaban se caracterizaban por una sobreocupación de la inclusa y de las nodrizas internas, que conllevaba el uso de forma complementaria de la lactancia artificial, casi siempre letal. Ocurrió en la inclusa de Florencia, en el que la falta de amas externas producía unos incrementos significativos en las tasas de mortalidad de los expósitos (Viazzo, Bortolotto y Zanotto, 1994).

17. En Italia se prefería a mujeres de zonas rurales ya que se consideraba que eran lugares más sanos para los niños. Las mujeres que solicitaban ser nodrizas eran examinadas para comprobar su aptitud para la lactancia y aportaban documentación, un certificado de buena conducta suscrito por el párroco, partida de defunción del hijo biológico o destete a una edad adecuada y reconocimiento médico que acreditara el estado de salud general y la situación nutricional (Kertzer, Single y White, 1999).

18. Reglamento de régimen interior de los Establecimientos Reunidos de Toledo. Capítulo III. Toledo; 1902, p. 7.

19. Reglamento de régimen interior de los Establecimientos Reunidos de Toledo. Capítulo III. Toledo; 1902, p. 7.

20. Reglamento de régimen interior de los Establecimientos Reunidos de Toledo. Capítulo III. Toledo; 1902, p. 7.

21. Disposiciones similares se establecieron en otras inclusas españolas como la de Madrid (Vidal, 1995; p. 99) y la de Guipúzcoa (Valverde, 1994, pp. 169-173).
22. Aunque los datos no son estrictamente comparables, porque los trabajos mencionados analizan la mortalidad en la inclusa teniendo en cuenta todas las modalidades de ingreso, pueden ser una referencia para nuestro estudio. L. Valverde ofrece cifras de mortalidad en la inclusa de Pamplona del $47,5 \%$ para el período $1890-1894$ y del $47,1 \%$ en la de Guipúzcoa en 1900 (Valverde, 1994). En el estudio de A. Uribe sobre la inclusa de Navarra en el periodo 1900-1904 la mortalidad infantil se eleva al $62,2 \%$, resultando este aumento porque incluye la mortalidad perinatal (Uribe-Etxebarría, 1996). M. Esteban presenta una mortalidad del 433 $\%$ para la inclusa de Salamanca en 1898, elevándose hasta el 622 \%o en la hijuela de Ciudad Rodrigo (Esteban, 1991). A. Rodríguez da cifras de la inclusa de Pontevedra, Oviedo y Barcelona entre 1900-1903, aunque nos centraremos en la de 1903: $385 \%$ para Pontevedra, $406 \%$ para Oviedo y 515 \% en Barcelona (Rodríguez, 2008). Bárbara Revuelta estima que en la inclusa de Madrid esta tasa se situaba en torno al $800 \%$ entre 1900-1920 para descender hasta el $600 \%$ en el decenio posterior (Revuelta, 2011).

23. Bárbara Revuelta en su tesis doctoral sobre la inclusa de Madrid en los primeros treinta años del siglo XX, estima que la mortalidad infantil para el periodo se aproximaba al $700 \%$, aunque había inclusas españolas con un número modesto de ingresos anuales (inferior a 100) que mantenían tasas superiores al $500 \%$ o (Revuelta, 2011, p. 395). Igualmente, la inclusa de Barcelona a principios de siglo mantenía unas cifras cercanas al $400 \%$.

24. Esta situación se repetía en países como Gran Bretaña, pues en 1907 la mortalidad posneonatal de la población general era del $132 \%$, mientras que en las workhouses (instituciones asilares que acogían niños) aumentaba hasta el 286-392 \%o (Marks, 1993). En Italia, la mortalidad en las inclusas era elevada pero estaba sometida a oscilaciones relacionadas con el mayor número de abandonos de unos años a otros y en la accesibilidad para reclutar y pagar a las amas externas (Kertzer, Single y White, 1999). Entre 1897 y 1904 se estima que la inclusa de Florencia tenía una mortalidad entre el 280-340 \%o, sufriendo un repunte durante los años de la Primera Guerra Mundial (Viazzo, Bortolotto y Zanotto, 1994). Sin embargo, Fuchs cifra la mortalidad infantil en la inclusa de París en torno al $100 \%$ a principios del siglo XX (Fuchs, 1984).

25. Datos similares aporta Sanz Gimeno sobre la tasa de mortalidad infantil en la población española. 180,2 \%o en 1906 que se reduce a 119,6 \%o en 1932 (Sanz, 2001, p. 137). Otros autores presentan cifras coincidentes para el período de estudio: 186,6 \%o en 1906 y 124 \%o en 1930 (Dopico y Reher, 1998, pp.32-36).

26. En la inclusa de Mallorca hay diferencia real entre la mortalidad infantil y la juvenil, pues la segunda es inferior a la primera, mientras que en la Madrid ambos indicadores ofrecen cifras similares. (Pujades, 2008, pp. 442-43; Revuelta, 2011, p. 404).

27. Dada la dureza del régimen patológico al que se veían expuestos al principio de su vida, sería esperable la presencia 
de efectos selectivos (que habrían acabado con los más débiles) o de un efecto de acumulación de daños que los haría más sensibles a nuevas enfermedades. La perspectiva del curso de la vida y de los efectos selectivos o de daño permanente a causa de enfermedades o experiencias desfavorables tempranas a la salud en el contexto de la vida de una persona, ha sido estudiada por varios autores en contextos contemporáneos e históricos (Bengtsson y Mineau, 2009; Gagnon y Mazan, 2009; Kuh y Ben-Shlomo, 1997; Smith, Mineau, Garibotti, Kerber, 2009)

28. En el período analizado, los expósitos alojados en la inclusa de Toledo tardaron un promedio de 40 días en salir a lactar con nodrizas externas, aunque esta cifra se debe tomar con cautela al englobar los valores extremos de estancia en la institución, cuyo límite inferior fue de 1 día y el superior de 320 días. La estancia en la institución era muy variable y dependía esencialmente de las peticiones para lactar niños que realizaban las amas. Libros de Lactancias y Destetes. A.D.P.T.

29. Varios historiadores de la medicina señalan que el significado de la terminología médica histórica no puede ser capturada por términos científicos actuales y cuestionan la posibilidad de realizar diagnósticos retrospectivos. (Arrizabalaga, 1993; Kunitz, 1999).

30. Era común para gran parte del continente europeo que la mortalidad que afectaba a cada tramo de edad estuviese causada por las mismas enfermedades. En un estudio sobre la mortalidad infantil en la Escocia rural (1845-1945) se sostiene que durante el primes mes de vida muchos niños morían de debilidad congénita, mientras que eran las infecciones atribuidas a malas condiciones ambientales las causantes de gran parte de las muertes hasta el año de vida (Blaikie, 1998).

\section{BIBLIOGRAFÍA}

Álvarez, León C. (1980), Marginación social y mentalidad en Andalucía Occidental. Expósitos en Sevilla (1613-1910). Sevilla, Junta de Andalucía.

Arenal, Concepción (1861), La beneficencia, la filantropía y la caridad. Madrid, Imprenta del Colegio de Sordomudos y Ciegos.

Arrizabalaga, Jon (1993), "Diseases as causes of death in early modern European medicine: Some historiographic considerations". Comunicación presentada en el Seminario History of Registration of Causes of Death Conference, Bloomington.

Bardet, J. P.; Dufour, C. y Rendard, J. (1997), "The Death of Foundlings: A Tragedy in Two Acts". En: Bideau, Alain; Desjardins, Bertrand y Pérez Brignoli, Héctor, Infant and Child Mortality in the Past. Oxford, Clarendon Press, pp. 245-261.

Barona, Josep L. (2004), “El Consejo Superior de Protección a la Infancia y Represión de la Mendicidad (1904-1914). Su ideo-
31. El mismo patrón se daba en la mortalidad para este grupo de edad en el conjunto de la población española (Sanz y Ramiro, 2002, p.167).

32. La mortalidad infantil en España se atribuye en gran medida a causas infecciosas para el conjunto de la población y se caracterizaba por la fuerte incidencia de enfermedades de transmisión aérea y por agua y alimentos (Sanz y Ramiro, 2002).

33. Estudios sobre mortalidad en el campo de la demografía histórica muestran la dificultad de conocer con precisión los procesos patológicos reales que se habían dado bajo esa etiqueta diagnóstica. Se ha comprobado que el diagnóstico "eclampsia" no solo representaba patologías como la epilepsia sino otras enfermedades infeccionas que podían provocar convulsiones febriles. Igualmente se puso de moda utilizar el término "atrepsia" en vez de otros como "enteritis", aunque por el comportamiento estacional de la enfermedad se refería a esta última (Bernabeu, Ramiro, Sanz y Robles, 2003).

34. Bárbara Revuelta en su tesis decide incorporar la atrepsia, la toxicosis y la atrofia a una categoría que denomina "causas de muerte relacionadas con la alimentación" en las que no distingue si el proceso es infeccioso o no (Revuelta, 2011, p.432).

35. Resultados similares muestra el trabajo de Bárbara Revuelta sobre la inclusa de Madrid para el periodo citado, ya que estima que la probabilidad de morir más elevada para el período posneonatal se relacionaba con el agua, los alimentos y la nutrición (Revuelta, 2011).

36. Tasas similares estima Bárbara Revuelta para la inclusa de Madrid (Revuelta, 2011, p. 443).

logía social y sanitaria". En: Perdiguero Gil, Enrique (ed.), Salvad al niño. Estudios sobre la protección a la infancia en la Europa Mediterránea a comienzos del siglo XX. Valencia, Guada Litografía, pp. 121-153.

Bernabeu, Josep; Ramiro, Diego; Sanz, Alberto y Robles, Elena (2003), "El análisis histórico de mortalidad por causas. Problemas y soluciones", Revista de Demografía Histórica, 21 (1), pp. 167-193.

Bengtsson, Tommy y Mineau, Geraldine P. (2009), "Early-life effects on socio-economic performance and mortality in later life: A full life-course approach using contemporary and historical sources", Social Science \& Medicine, 68, pp. 1561-1564.

Blaikie, Andrew (1998), "Scottish Illegitimacy: Social Adjustment or Moral Economy?", Journal of Interdisciplinary History, 29 (2), pp. 221-241. 
Blaikie, Andrew (1998), "Infant survival chances, unmarried motherhood and domestic arrangements in Rural Scotland, 1845-1945", Local Population Studies, 60 , pp. 34-46.

Bolufer, Mónica (1992), "Actitudes y discursos sobre la maternidad en la España del siglo XVIII: la cuestión de la lactancia", Historia Social, 14, pp. 3-22.

Chacón, Francisco; Elgarrista, Rosa y Fresneda, Rafael (1987), "Mercenarismo ¿̇mito o realidad? Análisis del comportamiento de las amas de cría en el reino de Murcia (siglos XVII-XVIII)". En: Chacón, Francisco (ed.), Familia y sociedad en el Mediterráneo occidental: siglos XV-XIX. Universidad de Murcia, pp. 93-116.

Dopico, Fausto y Reher, David S. (1998), El declive de la mortalidad en España, 1860-1930. Colección Monografías AEDH. Prensas universitarias de Zaragoza.

Esteban, Mariano (1991), De la beneficencia a la previsión. La acción social en Salamanca (1875-1898). Diputación de Salamanca.

Fernández, María (1988), Expósitos en Salamanca a comienzos del siglo XVIII. Salamanca, Diputación de Salamanca.

Fuchs, Rachel G. (1984), Abandoned children: foundlings and child welfare in nineteenth century France. State University of New York Press.

Fuente, M. Prado de la (2000), Marginación y pobreza en la Granada de la 2a mitad del siglo XVIII: los niños expósitos. Universidad de Granada.

Galicia Pinto, Isabel (1985), La Real Casa Hospicio de Zamora: asistencia social a marginados (1798-1850). Diputación Provincial de Zamora.

Gagnon, Alain y Mazan, Ryan (2009), "Does exposure to infectious diseases in infancy affect old-age mortality?", Social Science \& Medicine, 68 (9), pp. 1609-16.

García Galán, Sonia (2011), "De las prácticas tradicionales a la supervisión médica en el ejercicio de la maternidad. Asturias 1900-1931", Dynamis, 31 (1), pp. 131-157.

Gómez, Trino (1997), El hospital real de Santa María Magdalena y la casa de expósitos. Almería, Instituto de Estudios Almerienses.

Gómez Redondo, Rosa (1992), La mortalidad infantil española en el siglo XX, Madrid, Centro de Investigaciones Sociológicas: Siglo XXI.

Kertzer, David I. (1991), “Gender Ideology and Infant Abandonment in Nineteenth-Century Italy", The Journal of Interdisciplinary History, 22 (1), pp. 1-25.

Kertzer, David I. (1999), "Syphilis, Foundlings and Wetnurses in Nineteenth-century Europe", Journal of Social History, 32 (3), pp. 589-602

Kertzer, David I., Single, Wendy y White, Michael J. (1999), "Childhood mortality and quality of care among abandoned children in nineteenth-century Italy", Population Studies, 53(3), pp. 303-315

Kuh, Diana y Ben-Shlomo, Yoav (1997), Life Course Approach to Chronic Disease Epidemiology. Tracing the origins of illhealth from early to adult life. Oxford University Press.

Kunitz, Stephen J (1999), "Premises, premises: Comments on the comparability of classifications", Journal of the History of Medicine, 54, pp. 226-240.

Luddy, Maria (2001), “Moral Rescue and Unmarried Mothers in Ireland in the 1920s", Women's Studies, 30 (6), pp. 797-817.

Martín-Espinosa, Noelia M. (2012), Madres que abandonan: niños expósitos en la inclusa de Toledo (1900-1930), tesis doctoral defendida en el Departamento de Historia Contemporánea. Universidad de Castilla-La Mancha.

Marks, Lara (1993), “Medical care for pauper mothers and their infants. Poor law provision and local demand in east London, 1870-1929", The Economic History Review, 46 (3), pp. 518-542.

Morel, France (2010), "Images de nourrices dans la France des XVIIle et XIXe siècles", Paedagogica Historica, 46 (6), pp. 803-817.

Ortega Munilla, José (1883), "La nodriza-ogro", La madre y el niño, pp. 140-141.

Perdiguero, Enrique y Robles, Elena (2004), “La protección a la infancia y la Sociedad Española de Higiene". En: Perdiguero Gil, Enrique, Salvad al niño. Estudios sobre la protección a la infancia en la Europa Mediterránea a comienzos del siglo XX. Valencia, Guada Litografía, pp. 93-122.

Pujades, Joana (2008), La modernització d'un sistema demogràfic urbà: Anàlisi e la mortalitat infantil i juvenil a la ciutat de Palma (1838-1960), Tesis doctoral defendida en el Departamento de Historia Contemporánea. Universitat de les Illes Balears.

Revuelta, Bárbara A. (2011), Los usos de la inclusa de Madrid, mortalidad y retorno a principios del siglo XX (1890-1935), tesis doctoral defendida en el Departamento de Historia Contemporánea. Universidad Complutense de Madrid.

Rodríguez Martín, Ana M. (2005), Las mujeres y la beneficencia en Barcelona en la segunda mitad del siglo XIX. La Casa Provincial de Maternidad y Expósitos de Barcelona, 1853-1903, Tesis doctoral defendida en el Departamento de Historia Contemporánea. Universidad de Barcelona.

Rodríguez Martín, Ana M. (2008), “Una estrategia de supervivencia familiar en Barcelona, en la segunda mitad del siglo XIX", Nuevo Mundo Mundos Nuevos [en línea],Coloquios. Disponible en: https://nuevomundo.revues.org/22322, [Consultado el 15-12-2013].

Rodríguez Ocaña, Esteban (1985), "Los consultorios de lactantes y gotas de leche en España”, Jano, 663, pp. 1066-1077.

Rodríguez Ocaña, Esteban (2001), "La Higiene Infantil”. En: Atenza Fernández, J. y Martínez Pérez, J., El centro secun- 
dario de Higiene Rural de Talavera de la Reina y la Sanidad española de su tiempo. Toledo, Junta de Comunidades de Castilla La Mancha.

Sanz, Alberto (2001), "Infancia, mortalidad y causas de muerte en España en el primer tercio del siglo XX (1906-1932)", Reis: revista española de investigaciones sociológicas, 95, pp. 129-154.

Sanz, Alberto y Ramiro, Diego (2002), "La caída de la mortalidad en la España interior, 1860-1960. Un análisis de las causas de la muerte", Cuadernos de Historia Contemporánea, 24, pp. 151-188.

Sarasúa, Carmen (1994), Criados, nodrizas y amos. El servicio doméstico en la formación del mercado de trabajo madrileño, 1758-1868. Madrid, Siglo XXI.

Smith, Ken R.; Mineau, Geraldine P.; Garibotti, Gilda y Kerber, Richard (2009), "Effects of childhood and middle-adulthood family conditions on later-life mortality: Evidence from the Utah Population Database, 1850-2002", Social Science \& Medicine, 68 (9), pp. 1649-1658.

Sussman, George D. (1975), "The Wet-nursing business in nineteenth-century France", French Historical Studies, 9 (2), pp.304-328.

Tarifa, Adela (1994), Pobreza y asistencia social en la España moderna: la Cofradía de San José y Niños Expósitos de Úbeda (siglos XVII y XVIII). Jaén, Instituto de Estudios Giennenses.
Testón, Isabel; Sánchez, Rocío y Blanco, José Pablo (2004), “Mi padre y mi madre me abandonaron, pero el Señor me acogió. Expósitos y lactancia mercenaria en la Puebla de Guadalupe". En: García Fernández, Máximo y Sobaler Seco, Mạ Ángeles (coord.), Estudios en homenaje al profesor Teófanes Egido. Universidad de Valladolid, pp. 381-401.

Torrubia, Eulalia (2004), Marginación y pobreza: expósitos de Salamanca (1794-1825). Diputación Provincial de Salamanca.

Uribe-Etxebarría, Aránzazu (1996), Marginalidad protegida: mujeres y niños abandonados en Navarra 1890-1930. Bilbao, Universidad del País Vasco.

Valverde, Lola (1994), Entre el deshonor y la miseria. Infancia abandonada en Guipúzcoa y Navarra. Siglos XVIII y XIX. Bilbao, Universidad del País Vasco.

Viazzo, Pier P.; Bortolotto, María y Zanotto, Andrea (1994), "Child care, infant mortality and the impact of legislation: the case of Florence's Foundling Hospital, 1840-1940", Continuity and Change, 9 (2), pp. 243-269.

Vidal, Florentina (1995), Bordes y bastardos: una historia de la inclusa de Madrid. Madrid, Compañía Literaria.

Wolf, Jacqueline H. (1999), “'Mercenary Hirelings' or 'A Great Blessing?': doctors' and mothers' conflicted perceptions of wet nurses and the ramifications for infant feeding in Chicago, 1871-1961", Journal of Social History, 33 (1), pp. 97-120. 